\title{
p53/microRNA-374b/AKT1 regulates colorectal cancer cell apoptosis in response to DNA damage
}

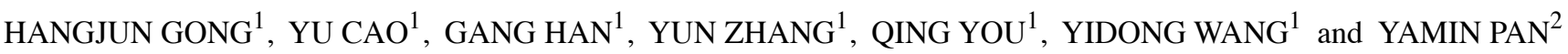 \\ ${ }^{1}$ Department of Gastrointestinal Surgery and ${ }^{2}$ The First Department of Endoscopy, Shuguang Hospital \\ Affiliated to Shanghai University of Traditional Chinese Medicine, Shanghai 201203, P.R. China
}

Received January 11, 2017; Accepted March 7, 2017

DOI: 10.3892/ijo.2017.3922

\begin{abstract}
Colorectal cancer (CRC) has a rising morbidity worldwide and its resistance to chemotherapy has been observed in clinical treatment. Tumor suppressor p53 is well-studied in CRC, but little is known about its effects during DNA damage of CRC cells. This study was aimed at uncovering potential mechanisms of p53 regarding microRNA-374b and v-akt murine thymoma viral oncogene homolog 1 (AKT1) during DNA damage of CRC cells. CRC cells HCT116 and HT29 were transfected with p53-specific small interfering RNA (siRNA), p53 overexpression vector or miR-374b inhibitor, and then treated with $10 \mu \mathrm{M}$ bleomycin (BLM) for $24 \mathrm{~h}$ to induce DNA damage. Primary (pri), precursor (pre) and mature miR-374b levels were quantified by qRT-PCR. AKT1 and p53 protein levels were detected by western blotting. Cell apoptosis changes were assessed by flow cytometry. AKT1 mRNA was detected to be induced by BLM treatment $(\mathrm{P}<0.05)$, but its protein level was strongly inhibited. Knockdown of p53 reversed the inhibition of AKT1 protein by BLM. Overexpression of p53 in p53-knockout HCT116 and HT29 cells upregulated the AKT1 regulator miR-374b $(\mathrm{P}<0.05)$, and knockdown of p53 reversed the induction of miR-374b by BLM. qRT-PCR suggested that besides mature miR-374b, p53 could also promote pre-miR374b level $(\mathrm{P}<0.05)$, rather than pri-miR-374b. Moreover, inhibition on miR-374b relieved the suppressed AKT1 protein, and reduced cell apoptosis induced by BLM. These data depict the $\mathrm{p} 53 / \mathrm{miR}-374 \mathrm{~b} / \mathrm{AKT} 1$ signaling that may regulate BLM-induced apoptosis in CRC cells, thus facilitating to improve the outcome of chemotherapy in CRC.
\end{abstract}

Correspondence to: Dr Hangjun Gong, Department of Gastrointestinal Surgery, 3B Ward, Eastern Branch, Shuguang Hospital Affiliated to Shanghai University of Traditional Chinese Medicine, 528 Zhangheng Road, Shanghai 201203, P.R. China

E-mail: gonghangjun111@126.com

Key words: colorectal cancer, p53, miR-374b, v-akt murine thymoma viral oncogene, DNA damage

\section{Introduction}

Colorectal cancer (CRC) is a common malignancy in the colon or rectum, which has a high morbidity and mortality worldwide. In the United States, CRC has become the third most common cancer among the top leading causes of cancer death (1). Great efforts have been made to improve prognosis and treatment outcome of CRC, such as the flexible sigmoidoscopy screening method, various prognostic biomarkers and histopathological classification criteria (2-4). Current therapy for CRC relies on surgery and adjuvant radiotherapy for rectal cancer or chemotherapy for colon cancer (5). Unfortunately, the resistance of CRC cells to chemotherapy impedes the expected outcome of CRC treatment $(6,7)$.

Chemotherapeutics usually utilize the cytotoxicity from synthetic chemical drugs to inhibit cancer cell growth and accelerate apoptosis. For instance, bleomycin (BLM) is widely applied in cancer treatment for its induction of DNA damage in proliferating cells (8). Several factors are revealed to strongly affect the resistance of cancer cells to chemotherapy, including v-akt murine thymoma viral oncogene (AKT), whose inhibitor reduces resistance to chemotherapeutic drugs (9). Moreover, some microRNAs are considered to be involved in the regulation of drug resistance, such as microRNA-374b (miR-374b), which can restore cisplatin sensitivity of pancreatic cancer cells (10), suggesting avenues for enhancing effects of chemotherapy.

Substantial evidence supports that tumor protein p53 is a tumor suppressor in various diseases including CRC. Expression of p53 can be induced by anticancer agents to speed up CRC cell apoptosis and improve general health of patients $(11,12)$. Numerous studies have shown the participation of p53 in cancer cell response to DNA damage (13-15), however, there are still much needs to be revealed about the specific function and mechanism of p53 in CRC cells in response to DNA damage.

The present study aimed to elucidate the mechanism of p53 in regulating CRC cell apoptosis in response to DNA damage. The above-mentioned factors in chemotherapy, AKT1 and miR-374b were also involved in this study in order to depict a more detailed frame for p53 mechanism. CRC cell line HCT116 and HT29 were treated by BLM to induce DNA damage. Cell transfection with p53-specific small interfering RNA (siRNA), p53 overexpression vector or miR-374b inhibitor 
were performed to reveal the effect of these factors. These experiments were expected to provide general knowledge for p53 and its related factors in CRC cells upon DNA damage.

\section{Materials and methods}

Cell culture. Human colorectal cancer cell lines HCT116 and HT29 were purchased from ATCC (Manassas, VA, USA). Knockout of p53 was performed by Genloci Biotechnologies

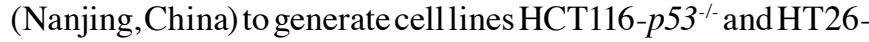
$p 53^{--}$. The cells were separately cultured in ATCC-formulated McCoy's 5A medium modified (ATCC) supplemented with $10 \%$ fetal bovine serum (FBS). The medium was changed every three days and the cells were incubated at $37^{\circ} \mathrm{C}$ in humidified atmosphere with $5 \% \mathrm{CO}_{2}$.

Cell transfection. The complete coding sequence of human p53 (GenBank accession no. AB082923) was inserted into pcDNA3-flag vector (Novagen, Darmstadt, Germany), and the correct plasmid was screened by sequencing. The specific siRNA for p53 (si-p53), the negative control (si-control), miR374b inhibitor and inhibitor control were produced by Ribobio (Guangzhou, China). Cell transfection was performed with the assistance of Lipofectamine-2000 (Invitrogen, Carlsbad, CA, USA) according to the manufacturer's instructions. Before transfection, the cells were seeded in FBS-free medium in 24-well plates $\left(2 \times 10^{5}\right.$ cells per well) to reach the confluence of approximately $90 \%$. The transfection complex was prepared and added to each well to a certain concentration $(1 \mu \mathrm{g} / \mathrm{ml}$ for Flag-p53 and blank vector, $50 \mathrm{nM}$ for si-p53 and si-control, and $100 \mathrm{nM}$ for miR-374b inhibitor and inhibitor control). The plates were incubated at $37^{\circ} \mathrm{C}$ for $24 \mathrm{~h}$ and then cells were used in further treatment and detection.

BLM treatment. BLM (Invitrogen) was dissolved in phosphatebuffered saline (PBS) before use. After $24 \mathrm{~h}$ of incubation for transfection, the cells were incubated in fresh medium containing BLM of $10 \mu \mathrm{M}$, and then incubated for another $24 \mathrm{~h}$ (15). Cells with PBS treatment were set as control groups. After BLM treatment, cells of each group were collected for further analysis.

Cell apoptosis assay. Four groups of cells were detected for cell apoptosis: cells transfected with miR-374b inhibitor + BLM treatment, cells transfected with inhibitor control + BLM treatment, and two control groups without BLM treatment. Cell apoptosis assay was performed by Annexin V-fluorescein isothiocyanate (FITC) and propidium iodide (PI) staining followed by flow cytometry. Annexin V-FITC Apoptosis Detection Kit I (Univ-Bio, Shanghai, China) was used to stain the cells according to the manufacturer's instructions. Briefly, the density of cells was adjusted to $1 \times 10^{6} / \mathrm{ml}$, and $200 \mu \mathrm{l}$ was used for each assay. The cells were washed in PBS two times and resuspended in $100 \mu \mathrm{l}$ binding buffer with $2 \mu \mathrm{l}$ Annexin V-FITC, and then incubated in the dark on ice for $15 \mathrm{~min}$. PBS $(400 \mu \mathrm{l})$ and $1 \mu 1$ PI solution was added and the samples were immediately detected by a flow cytometry FACSCanto II (BD Biosciences, San Jose, CA, USA). FITC-positive and PI-negative cells were considered as apoptotic cells.
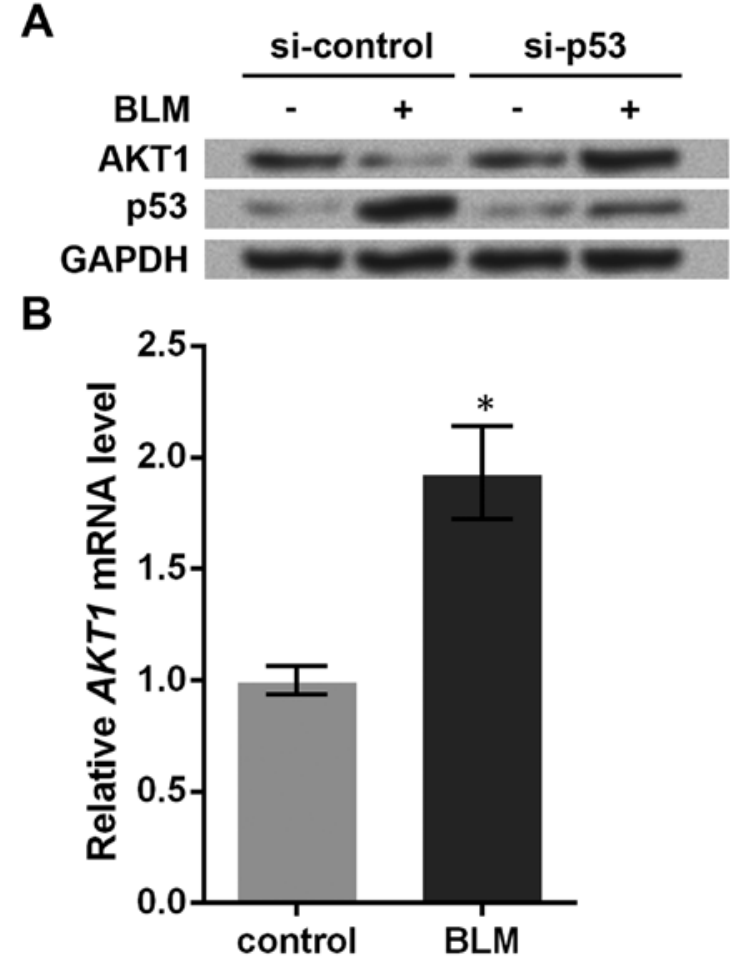

Figure 1. Knockdown of p53 abrogates the bleomycin (BLM)-suppressed v-akt murine thymoma viral oncogene homolog 1 (AKT1) protein level. Colorectal cancer HCT116 cells were transfected with the specific small interfering RNA for p53 (si-p53) or negative control (si-control), and then treated with BLM to induce DNA damage. (A) Western blot detection of the protein level changes of AKT1 and p53. GAPDH is an internal control. (B) qRT-PCR detectiom of the elevated $A K T 1$ mRNA level in untransfected HCT116 cells after BLM treatment. ${ }^{*} \mathrm{P}<0.05$ compared to the control group.

$q R T-P C R$. RNA samples of cells were isolated with TRIzol (Invitrogen) according to the manufacturer's instructions and purified with RNA purification kit (Tiangen, Beijing, China). The quantification of miRNAs were performed based on a previous study (16). Reverse transcription for pri-miRNAs was performed with $1 \mu \mathrm{g}$ RNA for each sample under the catalysis of SuperScript III Reverse Transcriptase (Invitrogen). Reverse transcription for pre-miRNAs and mature miRNAs were performed by One Step PrimeScript miRNA cDNA Synthesis (Takara, Dalian, China). qRT-PCR was conducted on QuantStudio 6 Flex Real-time PCR System (Applied Biosystems, Carlsbad, CA, USA), and the reaction conditions were: pre-denaturation at $95^{\circ} \mathrm{C}$ for $10 \mathrm{~min}, 40$ cycles of $95^{\circ} \mathrm{C}$ for $30 \mathrm{sec}, 64^{\circ} \mathrm{C}$ for $30 \mathrm{sec}$ and $72^{\circ} \mathrm{C}$ for $30 \mathrm{sec}$, followed by a melting curve of $95^{\circ} \mathrm{C}$ for $15 \mathrm{sec}, 60^{\circ} \mathrm{C}$ for $1 \mathrm{~min}$ and $95^{\circ} \mathrm{C}$ for $15 \mathrm{sec}$. Each reaction sample contained $20 \mathrm{ng}$ complementary DNAs and a pair of specific primers for mature miR-374b-5p (forward: 5'-CACTC CAGCT GGGAT ATAAT ACAAC CTGC-3' and reverse: 5'-TGGTG TCGTG GAGTC G-3'), premiR-374b (forward: 5'-CCTAT ATTAT GTTGG ACG-3' and reverse: 5'-CTGTG CCTGT TACTA TTATG-3'), pri-miR374b (forward: 5'-ACCAT CTGCT CTCGG TATG-3' and reverse: 5'-CCTGG AGTGG TGCTC CTCTG-3'), pre-miR-34 (forward: 5'-GGCCT CGACA CTCAC AAA-3' and reverse: 5'-GGTGT TGCAC GTCGT GAA-3') or pri-miR-34 (forward: 5'-GAGGC CTACG GCACC TGG-3' and reverse: 5'-GAGCG AAGTA GAAGG GAGAA-3'). Results were normalized by 
A

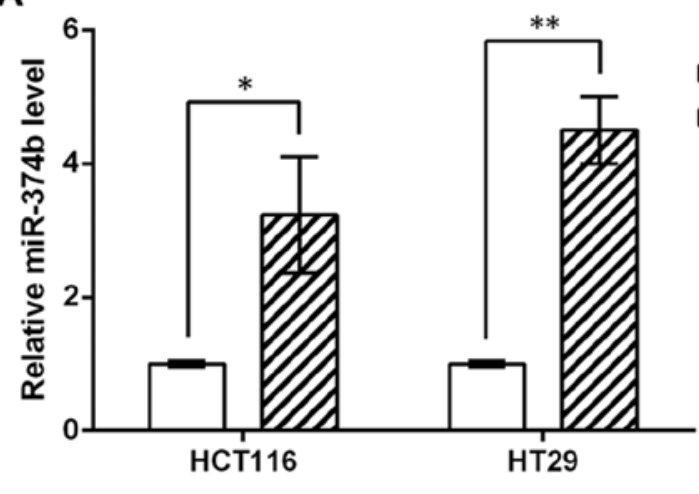

B

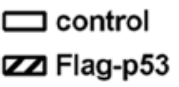

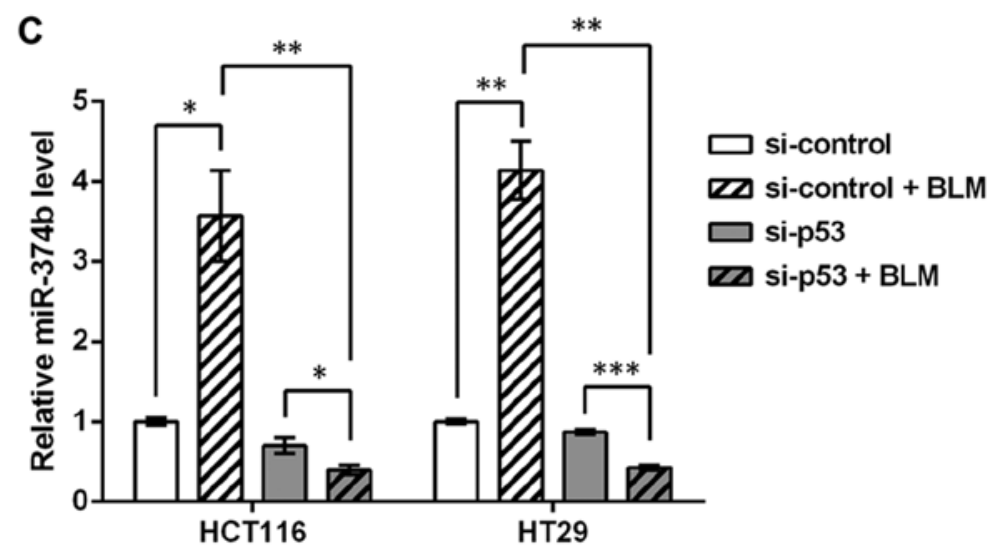

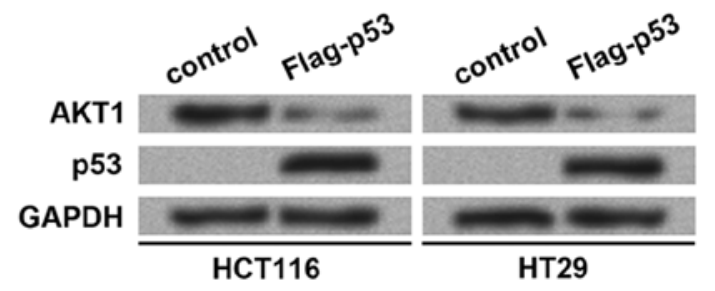

Figure 2. Knockdown of p53 abrogates the bleomycin (BLM)-induced miR-374b level in HCT116 and HT29 cells. p53\% HCT116 and HT29 cells were transfected with Flag-tagged p53 overexpression vector. Wild-type HCT116 and HT29 cells were transfected with the specific small interfering RNA for p53 (si-p53) or negative control (si-control), and then treated with BLM to induce DNA damage. (A) p53 overexpression induces miR-374b level in $p 53^{-/-}$HCT116 and HT29 cells. (B) Western blot detection of v-akt murine thymoma viral oncogene homolog 1 (AKT1) protein and p53 protein (by anti-Flag antibodies) in HCT116 and HT29 cells $\left(p 53^{-/}\right)$. GAPDH is an internal control. (C) qRT-PCR shows that BLM elevated miR-374b level in wild-type HCT116 and HT29 cells, but this effect is abrogated when knocking down $\mathrm{p} 53 .{ }^{*} \mathrm{P}<0.05,{ }^{* *} \mathrm{P}<0.01,{ }^{* * * *} \mathrm{P}<0.001$.

GAPDH (forward: 5'-GGTGA TCCTG GTGAA GGAGA-3' and reverse: 5'-CTTAA TGTGC CCGTC CTTGT-3') for primiRNAs or U6 (forward: 5'-CTCGC TTCGG CAGCA CATAT ACT-3' and reverse: 5'-ACGCT TCACG AATTT GCGTG TC-3') for miR-374b or pre-miRNAs. Similarly, AKT homolog 1 (AKT1) mRNA level was detected with its specific primers (forward: 5'-GAAGG TGAAG GTCGG AGTC-3' and reverse: 5'-GAAGA TGGTG ATGGG ATTTG-3') and normalized by $G A P D H$. Data were calculated with the $2^{-\triangle \Delta C t}$ method.

Western blotting. Protein samples from cells were isolated with M-PER mammalian protein extraction reagent (Thermo Scientific, Carlsbad, CA, USA) and quantified with Bio-Rad Protein Assay (Bio-Rad, Hercules, CA, USA). Protein was separated by sodium dodecyl sulfate-polyacrylamide gel electrophoresis and blotted to polyvinylidene fluoride membranes. The blots were blocked in $5 \%$ skim milk overnight at $4^{\circ} \mathrm{C}$, and then incubated in rabbit monoclonal antibodies against AKT1 or p53 (1:1000, ab32505 and ab32049, Abcam, Cambridge, UK) for $2 \mathrm{~h}$ at room temperature. Anti-GAPDH antibodies (1:1000, ab9485) were used as an internal control. After washed in PBS 3 times (5 min each time), the blots were incubated in horseradish peroxidase (HRP)-conjugated goat anti-rabbit secondary antibodies (1:2000, ab7090) for $1 \mathrm{~h}$ at room temperature. Signals were developed by EasyBlot ECL kit (Sangon Biotech, Shanghai, China) and the relative density was analyzed by ImageJ 1.49 (National Institutes of Health, Bethesda, MD, USA).

Statistical analysis. All experiments were performed in triplicate. Results are presented as mean \pm standard deviation. Data were analyzed with one-way analysis of variance (ANOVA) and t-test by SPSS 20 (IBM, New York, NY, USA). Difference between groups was considered significant at $\mathrm{P}<0.05$.

\section{Results}

p53 promotes miR-374b and inhibits $A K T 1$ protein in response to DNA damage. Since AKT has been found to be involved in regulation of cell sensitivity to chemotherapy, this study began with analyzing the relationship between p53 and AKT1 in HCT116 cells. Western blotting showed that BLM treatment decreased AKT1 protein and increased p53 protein (Fig. 1A). However, the effect of BLM treatment was abrogated and AKT1 protein was increased when p53 was knocked down, implying that the suppression of AKT1 by BLM treatment was dependent on p53.

The underlying mechanism between p53 and AKT1 is unknown. To solve this, we detected $A K T 1$ mRNA levels in HCT116 cells before and after BLM treatment, and found that BLM treatment could significantly upregulate $A K T 1$ mRNA 
A

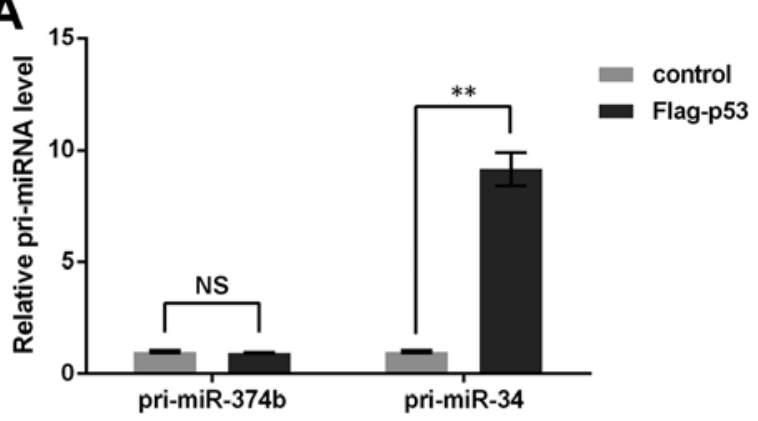

C

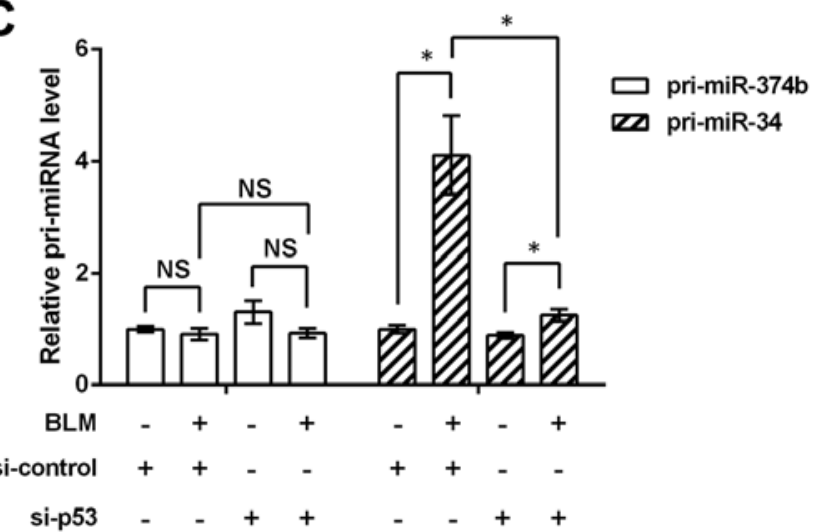

B

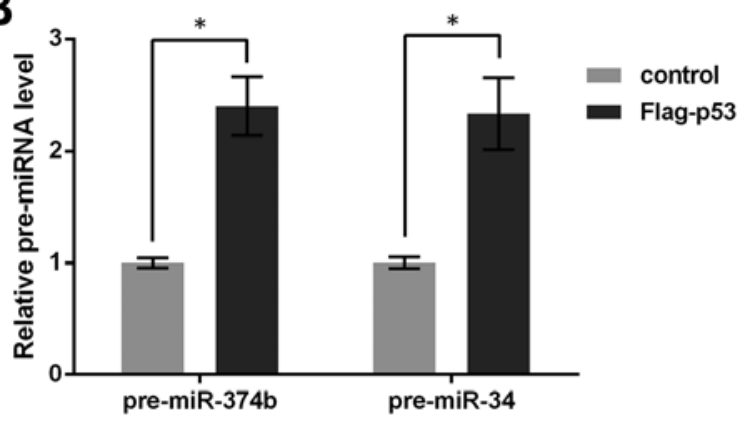

D

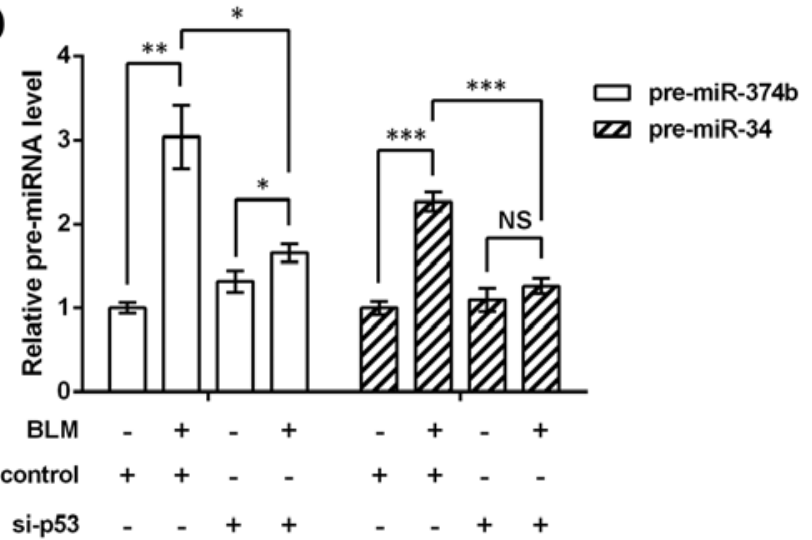

Figure 3. p53 regulates the processing of pri-miR-374b. Overexpression of p53 by its Flag-tagged vector and knockdown of p53 by its small interfering RNA (si-p53) were performed in $p 53^{-/ /}$and wild-type HCT116 cells, respectively. qRT-PCR was conducted to quantify miRNA levels. pri-miR-34 and pre-miR-34 were used as positive controls. (A) p53 overexpression does not affect pri-miR-374b level in $p 53^{-/}$HCT116 cells. (B) p53 overexpression induces pre-miR-374b in $p 53^{-/}$HCT116 cells. (C) Bleomycin (BLM) treatment or p53 knockdown does not affect pri-miR374b in wild-type HCT116 cells. (D) pre-miR-374b is induced by BLM treatment and then inhibited by 53 knockdown in wild-type HCT116 cells. ${ }^{*} \mathrm{P}<0.05,{ }^{* *} \mathrm{P}<0.01,{ }^{* * *} \mathrm{P}<0.001$. NS, not significant.

levels ( $\mathrm{P}<0.05$, Fig. 1B), which showed an opposite changing pattern compared to its protein level. In light of the disparity, it was likely that AKT1 was inhibited post-transcriptionally. Through searching TargetScanHuman 7.0 (17), we found AKT1 mRNA could be regulated by several miRNAs, among which miR-374b was related to cell chemosensitivity, and has been revealed as a direct up-stream factor of $A K T 1$ mRNA in C2C12 myoblasts (18). Thus, miR-374b was introduced into this study.

Factors p53, AKT1 and miR-374b were detected in the CRC cell lines HCT116 and HT29. Overexpression of p53 in the cell lines $\left(p 53^{-/}\right)$significantly induced miR-374b level $(\mathrm{P}<0.05$ or $\mathrm{P}<0.01$, Fig. $2 \mathrm{~A})$, and AKT1 protein expression was inhibited (Fig. 2B), which further suggested the reverse regulation between $\mathrm{p} 53$ and AKT1 protein. Then knockdown of p53 and BLM treatment were performed in wild-type cells. miR-374b level was significantly induced by BLM treatment $(\mathrm{P}<0.05$ or $\mathrm{P}<0.01$, Fig. $2 \mathrm{C})$, while it was significantly inhibited when p53 was knocked down $(\mathrm{P}<0.01)$, suggesting that the induction of miR-374b by DNA damage was also dependent on p53. Taken together, p53 might promote miR-374b to inhibit AKT1 protein expression in response to DNA damage in CRC cells.

p53 regulates the processing of pri-miR-374b. Next, the regulation of miR-374b by p53 was investigated. miRNA generation is composed of the processing of pri-miRNA to pre-miRNA, and pre-miRNA to mature miRNA (16), thereby the regulation of pri-miR-374b and pre-miR-374b by p53 was detected

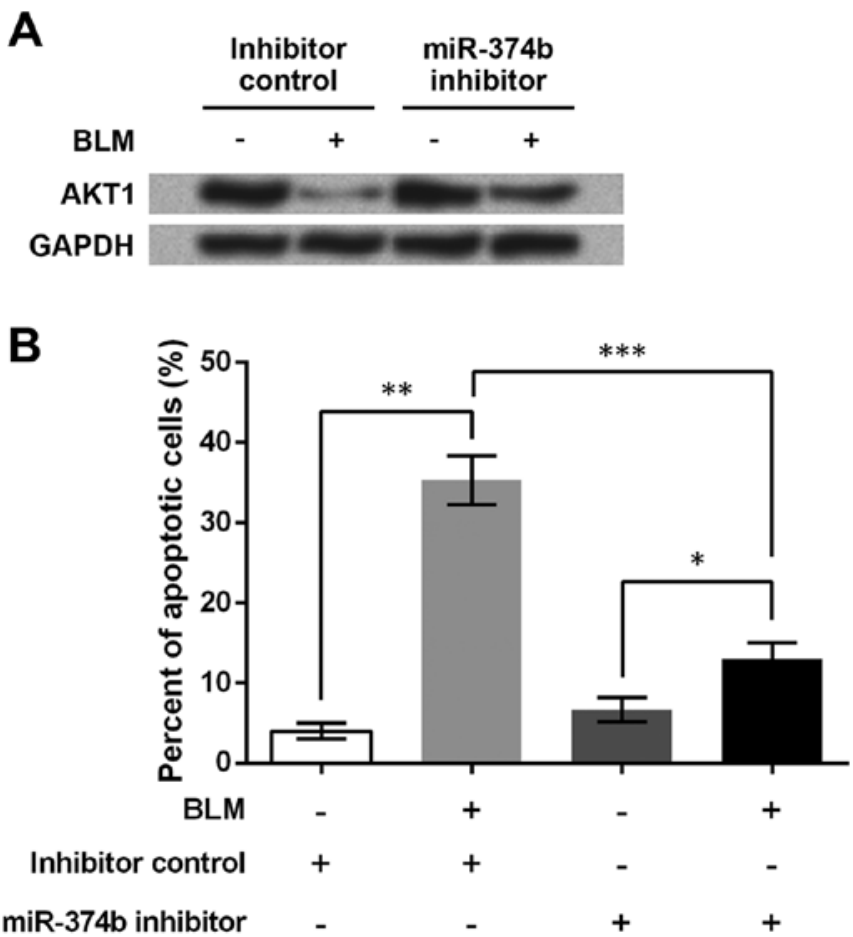

Figure 4. miR-374b inhibitor reduces the percentage of apoptotic cells in bleomycin (BLM)-induced HCT116. HCT116 cells were transfected with miR-374b inhibitor and then treated with BLM. (A) Western blot showing miR-374b inhibitor elevates v-akt murine thymoma viral oncogene homolog 1 (AKT1) protein that is suppressed by BLM treatment. (B) Quantified flow cytometry result showing cell apoptosis induced by BLM treatment is suppressed by miR-374b inhibitor. ${ }^{*} \mathrm{P}<0.05,{ }^{* * *} \mathrm{P}<0.01,{ }^{* * * *} \mathrm{P}<0.001$. 


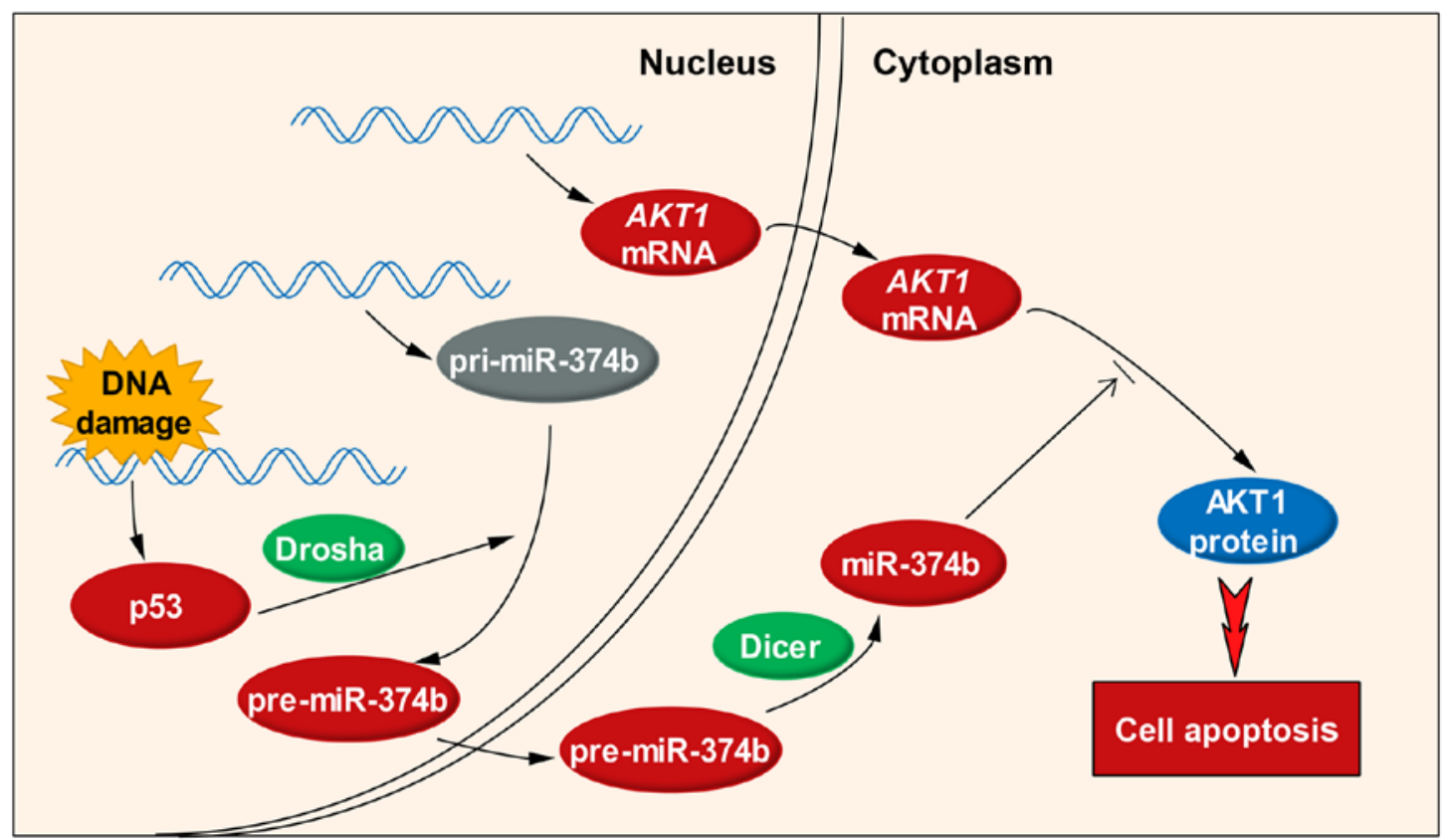

Figure 5. The cascade regulated by p53 in colorectal cancer (CRC) cells in response to DNA damage. DNA damage induces synthesis of p53 and v-akt murine thymoma viral oncogene homolog 1 (AKT1) mRNA, while it does not change the transcription of primary miR-374b (pri-miR-374b). The elevated p53 somehow stimulates the activity of RNase III enzyme Drosha to promote the processing of pri-miR-374b, thus generating more precursor miR-374b (pre-miR374b) and mature miR-374b (by RNase III enzyme Dicer). miR-374b directly targets AKT1 mRNA and inhibits the translation process, thereby the AKT1 protein level is decreased, contributing to CRC cell apoptosis. Red color indicates factors or processes that are promoted; blue color indicates factors that are suppressed; grey color indicates factors that are not changed in the cascade; green color indicates the RNase III enzymes Drosha and Dicer.

via qRT-PCR method, respectively. Moreover, miR-34 transcription is modulated by 53 , thus it was used as a positive control, and results showed both pri-miR-34 and pre-miR-34 were markedly upregulated by 53 in $p 53^{-/-}$HCT116 cells $(\mathrm{P}<0.05$ or $\mathrm{P}<0.01$, Fig. 3A and $\mathrm{B})$. Although pre-miR-374b was elevated by $\mathrm{p} 53(\mathrm{P}<0.05)$, no significant change was found in pri-miR-374b level, which implied that p53 did not impact pri-miR-374b.

Consistently, in the wild-type HCT116 cells with p53 knockdown and BLM treatment, it was found that pri-miR-374b was not affected by BLM or si-p53 (Fig. 3C), but pre-miR-374b could be upregulated by BLM treatment $(\mathrm{P}<0.01)$ and then suppressed by si-p53 ( $\mathrm{P}<0.05$, Fig. 3D). Together with the previous result that $\mathrm{p} 53$ could regulate mature $\mathrm{miR}-374 \mathrm{~b}$, it was possible that the regulation of miR-374b by p53 started with the processing of pri-miR-374b to pre-miR-374b, rather than the transcription of pri-miR-374b.

Inhibiting miR-374b reduces BLM-induced CRC cell apoptosis. Finally, the regulation of AKT1 and HCT116 cell apoptosis by miR-374b was analyzed. Western blotting showed that miR-374b inhibitor clearly relieved the BLM-induced inhibition on AKT1 protein (Fig. 4A), implying that miR-374b might help to suppress AKT1 expression. Since AKT1 mRNA was predicted to be a direct target of miR-374b, it was possible that miR-374b directly suppressed the translation of AKT1 protein.

HCT116 cell apoptosis was detected by flow cytometry after miR-374b transfection and BLM treatment. Changes in apoptotic cell percent indicated that BLM induced cell apoptosis $(\mathrm{P}<0.01$, Fig. 4B). Cell apoptosis was also elevated by $\mathrm{BLM}$ when miR-374b was inhibited $(\mathrm{P}<0.05)$, which, however, was significantly weakened because of the inhibited miR-374b $(\mathrm{P}<0.001)$. Thus suppressing miR-374b was able to inhibit HCT116 cell apoptosis upon DNA damage, which might be the result of the decreased sensitivity of HCT116 cells to BLM.

\section{Discussion}

Cancer cells respond differently to chemotherapy-induced DNA damage. In this study, BLM treatment induces p53 expression and upregulation of AKT1 mRNA, but AKT1 protein levels is downregulated in CRC HCT116 cells. Further analyses indicated p53 regulates the generation of pre-miR$374 \mathrm{~b}$, but did not affect pri-miR-374b level. When miR-374b is inhibited, AKT1 protein level arises and the HCT116 cell apoptosis is suppressed.

After pri-miRNAs are transcribed from the genome, they go through various procedures before generating mature miRNAs, two main steps among which are the cropping of pri-miRNAs to pre-miRNAs by RNase III enzyme Drosha and the dicing of pre-miRNAs to mature miRNAs by RNase III enzyme Dicer (19). The transcription of primiRNAs from the genome is also regulated by various factors in their promoter $(20,21)$. However, pri-miR-374b level was not affected by up- or down-regulation of p53 in HCT116 cells, suggesting that p53 has no dominant effect on the transcription of pri-miR-374b, although the possibility of its direct binding to the miR-374b promoter cannot be ruled out according to previous findings (22). pre-miR-374b and mature miR-374 levels could be promoted by p53, implying that p53 may promote the cropping step of miR-374b generation. Since p53 is a nuclear protein (23), it is less possible 
to regulate the dicing step. Moreover, existing evidence supports that p53 can modulate Drosha, and mutant p53 severely affected Drosha activity $(24,25)$. Thereby the results in this study demonstrate that p53 regulates the processing from pri-miR-374b to pre-miR-374b.

$A K T 1$ mRNA was conjectured to be a direct target of miR374b according to the prediction on TargetScanHuman 7.0 database. $A K T 1 \mathrm{mRNA}$ level was elevated, while its protein level is obviously downregulated in response to BLM treatment, suggesting a potential post-transcriptional regulation on AKT1 expression. miRNAs are powerful modulators of the translational status (26); moreover, this study indicated that AKT1 protein level was promoted by miR-374b inhibitor, implying a reverse regulation between the two factors. A previous study found evidence that miR-374b directly targets and inhibits $A K T 1$ mRNA, thus suppressing cell proliferation (27). Thus it is reasonable to speculate that miR-374b can also inhibit the translation from $A K T 1$ mRNA to AKT1 protein in HCT116 cells, which constitutes the downstream part of the p53 signaling.

Cell apoptosis was detected when miR-374b was inhibited, and miR-374b inhibitor was able to suppress the BLM-induced cell apoptosis, implying that miR-374b may promote HCT116 cell apoptosis. A similar role of miR-374b has been reported: miR-374b elevates chemotherapeutic agent-induced apoptosis in T-cell lymphoblastic lymphoma cells (27). Besides miR-374b, p53 and AKT1 are also greatly involved in the regulation of cell apoptosis, albeit playing different roles. As a tumor suppressor, p53 induces cell apoptosis through multiple pathways when DNA damage occurs or oncogene is overexpressed $(28,29)$, while AKT1 is a major AKT isoform that protects against apoptosis, as reported in various cell types including CRC cells (30-32). In this study, the induced changes in p53, miR-374b, AKT1 and cell apoptosis were consistent with existing studies on these factors, implying the p53, miR-374b and AKT1 may participate in the regulation of BLM-induced HCT116 cell apoptosis.

Based on the above results and discussion, a brief regulatory cascade (Fig. 5) containing p53, miR-374b and AKT1 can be depicted: in response to chemotherapy-induced DNA damage, a series of changes may happen in the nucleus, including the elevated expression of $\mathrm{p} 53$ and the promoted transcription of $A K T 1$ mRNA. The transcription of pri-miR-374b seems unaffected, but its processing to generate pre-miR-374b is accelerated, possibly due to the increased Drosha activity by p53. pre-miR-374b is transferred to the cytoplasm, where it is diced into mature miR-374b by the Dicer. Although AKTI mRNA level is upregulated, it is a potential direct target of miR-374b and its translation is suppressed, thus leading to the downregulation of AKT1 protein. Through this regulatory cascade, p53, miR-374b and AKT1 are capable of modulating HCT116 cell apoptosis in response to DNA damage. More details in the p53/miR-374b/AKT1 signaling are to be revealed in further research.

Resistance to chemotherapy of cancer cells can be modulated by p53 and AKT1. The role of p53 on cancer cell sensitivity to chemotherapy may be conflicting (33), but most studies demonstrated its promotive effects on the sensitivity of cancer cells to chemotherapy $(34,35)$. In addition, AKTl gene polymorphism influences neoadjuvant chemotherapeutic sensitivity of cancer cells (36). In this study, the p53/miR-374b/ AKT1 signaling was demonstrated to play potential roles in HCT116 cell apoptosis in response to BLM-induced DNA damage, which implies the possibility of modulating these factors to improve the sensitivity of CRC cells to chemotherapy.

In summary, this study uncovers the mechanism of p53 regarding the p53/miR-374b/AKT1 signaling in regulating HCT116 cell apoptosis in response to the BLM-induced DNA damage. These results demonstrate the p53/miR-374b/AKT1 signaling, as a potential significant modulator, improves the outcome of chemotherapy in treating CRC. Further efforts are need to reveal more effects of this signaling on cancer cells.

\section{References}

1. Siegel R, Desantis C and Jemal A: Colorectal cancer statistics, 2014. CA Cancer J Clin 64: 104-117, 2014.

2. Schoen RE, Pinsky PF, Weissfeld JL, Yokochi LA, Church T, Laiyemo AO, Bresalier R, Andriole GL, Buys SS, Crawford ED, et al; PLCO Project Team: Colorectal-cancer incidence and mortality with screening flexible sigmoidoscopy. N Engl J Med 366: 2345-2357, 2012.

3. Sobin LH, Gospodarowicz MK and Wittekind C (eds): TNM Classification of Malignant Tumours. John Wiley \& Sons, West Sussex, 2011.

4. Walther A, Johnstone E, Swanton C, Midgley R, Tomlinson I and Kerr D: Genetic prognostic and predictive markers in colorectal cancer. Nat Rev Cancer 9: 489-499, 2009.

5. Brenner H, Kloor M and Pox CP: Colorectal cancer. Lancet 383: 1490-1502, 2014

6. Dylla SJ, Beviglia L, Park IK, Chartier C, Raval J, Ngan L, Pickell K, Aguilar J, Lazetic S, Smith-Berdan S, et al: Colorectal cancer stem cells are enriched in xenogeneic tumors following chemotherapy. PLoS One 3: e2428, 2008.

7. Jensen NF, Stenvang J, Beck MK, Hanáková B, Belling KC, Do KN, Viuff B, Nygård SB, Gupta R, Rasmussen $\mathrm{MH}$, et al: Establishment and characterization of models of chemotherapy resistance in colorectal cancer: Towards a predictive signature of chemoresistance. Mol Oncol 9: 1169-1185, 2015.

8. Edhemovic I, Gadzijev EM, Brecelj E, Miklavcic D, Kos B, Zupanic A, Mali B, Jarm T, Pavliha D, Marcan M, et al: Electrochemotherapy: A new technological approach in treatment of metastases in the liver. Technol Cancer Res Treat 10: 475-485, 2011.

9. Martelli AM, Tazzari PL, Tabellini G, Bortul R, Billi AM, Manzoli L, Ruggeri A, Conte R and Cocco L: A new selective AKT pharmacological inhibitor reduces resistance to chemotherapeutic drugs, TRAIL, all-trans-retinoic acid, and ionizing radiation of human leukemia cells. Leukemia 17: 1794-1805, 2003.

10. Schreiber R, Mezencev R, Matyunina LV and McDonald JF: Evidence for the role of microRNA 374b in acquired cisplatin resistance in pancreatic cancer cells. Cancer Gene Ther 23: 241-245, 2016.

11. Li B, Zhao J, Wang C-Z, Searle J, He TC, Yuan CS and Du W: Ginsenoside Rh2 induces apoptosis and paraptosis-like cell death in colorectal cancer cells through activation of $\mathrm{p} 53$. Cancer Lett 301: 185-192, 2011

12. He ZY, Shi CB, Wen H, Li FL, Wang BL and Wang J: Upregulation of $\mathrm{p} 53$ expression in patients with colorectal cancer by administration of curcumin. Cancer Invest 29: 208-213, 2011.

13. Mirzayans R, Andrais B, Scott A and Murray D: New insights into p53 signaling and cancer cell response to DNA damage: Implications for cancer therapy. J Biomed Biotechnol 2012: $170325,2012$.

14. Ng KW, Khoo SP, Heng BC, Setyawati MI, Tan EC, Zhao X, Xiong S, Fang W, Leong DT and Loo JS: The role of the tumor suppressor p53 pathway in the cellular DNA damage response to zinc oxide nanoparticles. Biomaterials 32: 8218-8225, 2011.

15. Lee YS, Yoon S, Park MS, Kim JH, Lee JH and Song CW: Influence of p53 expression on sensitivity of cancer cells to bleomycin. J Biochem Mol Toxicol 24: 260-269, 2010.

16. Schmittgen TD, Lee EJ, Jiang J, Sarkar A, Yang L, Elton TS and Chen C: Real-time PCR quantification of precursor and mature microRNA. Methods 44: 31-38, 2008. 
17. Agarwal V, Bell GW, Nam JW and Bartel DP: Predicting effective microRNA target sites in mammalian mRNAs. eLife 4: e05005, 2015.

18. Ma Z, Sun X, Xu D, Xiong Y and Zuo B: MicroRNA, miR-374b, directly targets Myf6 and negatively regulates $\mathrm{C} 2 \mathrm{C} 12$ myoblasts differentiation. Biochem Biophys Res Commun 467: 670-675, 2015.

19. Suzuki HI and Miyazono K: Emerging complexity of microRNA generation cascades. J Biochem 149: 15-25, 2011.

20. Guil S and Esteller M: DNA methylomes, histone codes and miRNAs: Tying it all together. Int J Biochem Cell Biol 41: 87-95, 2009.

21. Marsico A, Huska MR, Lasserre J, Hu H, Vucicevic D, Musahl A, Orom U and Vingron M: PROmiRNA: A new miRNA promoter recognition method uncovers the complex regulation of intronic miRNAs. Genome Biol 14: R84, 2013.

22. Chang CJ, Chao CH, Xia W, Yang JY, Xiong Y, Li CW, Yu WH, Rehman SK, Hsu JL, Lee HH, et al: p53 regulates epithelialmesenchymal transition and stem cell properties through modulating miRNAs. Nat Cell Biol 13: 317-323, 2011

23. Muller PA and Vousden KH: p53 mutations in cancer. Nat Cell Biol 15: 2-8, 2013.

24. Jiang F-Z, He Y-Y, Wang H-H, Zhang HL, Zhang J, Yan XF, Wang XJ, Che Q, Ke JQ, Chen Z, et al: Mutant p53 induces EZH2 expression and promotes epithelial-mesenchymal transition by disrupting p68-Drosha complex assembly and attenuating miR-26a processing. Oncotarget 6: 44660-44674, 2015

25. Gurtner A, Falcone E, Garibaldi F and Piaggio G: Dysregulation of microRNA biogenesis in cancer: The impact of mutant p53 on Drosha complex activity. J Exp Clin Cancer Res 35: 45, 2016.

26. Engels BM and Hutvagner G: Principles and effects of microRNA-mediated post-transcriptional gene regulation. Oncogene 25: 6163-6169, 2006.

27. Qian D, Chen K, Deng H, Rao H, Huang H, Liao Y, Sun X, Lu S, Yuan Z, Xie D, et al: MicroRNA-374b suppresses proliferation and promotes apoptosis in T-cell lymphoblastic lymphoma by repressing AKT1 and Wnt-16. Clin Cancer Res 21: 4881-4891, 2015
28. Bates S and Vousden KH: Mechanisms of p53-mediated apoptosis. Cell Mol Life Sci 55: 28-37, 1999.

29. Kuribayashi K, Finnberg N, Jeffers JR, Zambetti GP and El-Deiry WS: The relative contribution of pro-apoptotic p53-target genes in the triggering of apoptosis following DNA damage in vitro and in vivo. Cell Cycle 10: 2380-2389, 2011.

30. Dihlmann S, Kloor M, Fallsehr C and von Knebel Doeberitz M: Regulation of AKT1 expression by beta-catenin/Tcf/Lef signaling in colorectal cancer cells. Carcinogenesis 26: 1503-1512, 2005.

31. Green BD, Jabbour AM, Sandow JJ, Riffkin CD, Masouras D, Daunt CP, Salmanidis M, Brumatti G, Hemmings BA, Guthridge MA, et al: Akt1 is the principal Akt isoform regulating apoptosis in limiting cytokine concentrations. Cell Death Differ 20: 1341-1349, 2013.

32. Tucka J, Yu H, Gray K, Figg N, Maguire J, Lam B, Bennett M and Littlewood T: Akt1 regulates vascular smooth muscle cell apoptosis through FoxO3a and Apaf1 and protects against arterial remodeling and atherosclerosis. Arterioscler Thromb Vasc Biol 34: 2421-2428, 2014.

33. Jackson JG, Pant V, Li Q, Chang LL, Quintás-Cardama A, Garza D, Tavana O, Yang P, Manshouri T, Li Y, et al: p53-mediated senescence impairs the apoptotic response to chemotherapy and clinical outcome in breast cancer. Cancer Cell 21: 793-806, 2012.

34. Chen GX, Zheng LH, Liu SY and He XH: rAd-p53 enhances the sensitivity of human gastric cancer cells to chemotherapy. World J Gastroenterol 17: 4289-4297, 2011.

35. Gu J, Tang Y, Liu Y, Guo H, Wang Y, Cai L, Li Y and Wang B: Murine double minute 2 siRNA and wild-type p53 gene therapy enhances sensitivity of the SKOV3/DDP ovarian cancer cell line to cisplatin chemotherapy in vitro and in vivo. Cancer Lett 343: 200-209, 2014

36. Guo L, Wu H, Zhu J, Zhang C, Ma J, Lan J and Xie X: Genetic variations in the PI3K/AKT pathway predict platinum-based neoadjuvant chemotherapeutic sensitivity in squamous cervical cancer. Life Sci 143: 217-224, 2015. 